\title{
Skeleton based Non rigid Shape Matching
}

\author{
Fariba AMINI ${ }^{1}$, Hossien EBRAHIMNEZHAD ${ }^{2, *}$ \\ ${ }^{1}$ Camputer Vision Researching Lab, Department of Electrical Engineering, Sahand University of \\ Technology, Tabriz, Iran \\ ${ }^{2}$ Camputer Vision Researching Lab, Department of Electrical Engineering, Sahand University of \\ Technology, Tabriz, Iran
}

\begin{abstract}
This paper offers a new feature for matching deformable objects based on skeleton. Effectiveness of the skeleton-based methods depends to a large extent on the quality and perfection of the extracted skeleton. When the skeleton is extracted, additional branches of which, with the shape contour segmentation, are omitted by DCE method. The pruned skeleton is robust to the complex deformations and noise. After the pruned skeleton is obtained, minimum distance of contour points of the shape from the skeleton is calculated. This distance almost is constant against the articulated changes which this feature is used for finding the corresponding points. In order to carry out matching, the suggested method is applied on the MPEG7 dataset. Results show that the proposed algorithm is highly effective for matching objects.
\end{abstract}

Keywords: optimized matching, shape matching, skeleton, shape contour, shape modeling, minimum distance.

\section{Introduction}

Deformable object matching is an important problem in computer vision. It has lots of applications in the field of object recognition, image registration, retrieval and object classification. Different methods have been proposed for shape matching up to now, most of which are either based on point or skeleton. Shape is used in many methods provided for matching. Shape, is a geometrical feature which can briefly explain the structure of an object in the picture. The issue common among all the offered method is the object transformation. As the figure (1) shows, a

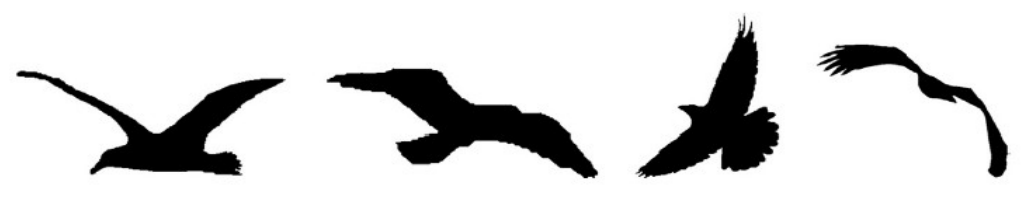

Fig. 1: An example of an object which has different shapes due to deformation

deformable object like a bird has different forms. Many methods such [1] has used the geometric-based shape descriptors. Others [2,3], has used the elastic matching algorithms. Among studies, skeleton is a powerful shape descriptor for the deformable objects because it consists of geometrical and topologic features of an object. That's why many skeleton finding algorithms have been proposed up to now. Shape contour was used in some previous methods for achieving the structure. In this case, additional branches are produced in the frame due to transformation or noise. In order to solve the problem, most of the proposed methods act in such a way as to obtain a skeleton which

\footnotetext{
*Email address: ebrahimmnezhad@sut.ac.ir
} 
has additional branches. Then, they omit the additional branches. Some features of the shape skeleton are: (1) to maintain the topologic information of the object; (2) not to change against small deformations; (3) to be unalterable against rotation and transfer; (4) to be close to the skeleton the human eye expects. Methodology used in this study includes all these features. Matching is done after the pruned skeleton is achieved. In a way that each point of the shape contour is attributed a minimum distance from the skeleton. This distance is almost remained constant against the object deformation. In the following, a brief review of the methods previously presented in terms of shape matching is discussed and the applied features are extracted. Some matching methods are based on contour $[4,5,6]$. These methods use the geometrical features like curvature and arc length. The Shape Context descriptor is another local feature in which the corresponding points are achieved by calculating the logarithmic polar diagram [1]. This method provides a context for other matching methods like [8,9]. Some other descriptors are based on skeleton. In these methods, how to obtain a skeleton is more important. More detailed the obtained skeleton; the more accurate matching is done. These methods are less sensitive against the shape deformations, in comparison to the contourbased ones, but are computationally more complex [10,11]. Graph shock is a descriptor based on the medial axis $[11,12]$. Skeleton and Shock graphs are widely used for object classification. The problem with these methods is that they don't do matching with a high accuracy. In the method [13] endpoints of the skeleton are considered as the important points. The corresponding final points are firstly obtained by studying the logarithmic polar diagram, and then, the corresponding parts of two segment are searched. By segmentation of the skeleton graph it is also possible to find the corresponding parts through studying the skeleton geometric paths [14,15], so that each part is between the final point and structure junction. Section 2 explains the proposed method, features extracted for matching and the function used. In section 3, experimental results of implementation on the MPEG7 dataset are evaluated. Section 4 outlines conclusion and future works for the research continuation.

\section{Proposed method}

In this paper, skeleton which consists of both topologic and geometric features is used for the shape matching. Centers of circles which are tangent on the contour in at least two points, forms the skeleton's points. Skeleton pruning and omitting its additional branches is done through the contour segmentation by Discrete Curve Evolution (DCE) method. After the pruned skeleton is extracted, contour's points distance from the structure is calculated which is almost remained constant under the shape deformation. This feature is used for matching deformable shapes which is studied in the following.

\subsection{Skeleton extraction}

Several studies have been proposed in order to achieve the skeleton, some of which are: using morphological operators [19], shortest distance of the shape's internal point from the boundary [17] and model of maximum circles tangent on

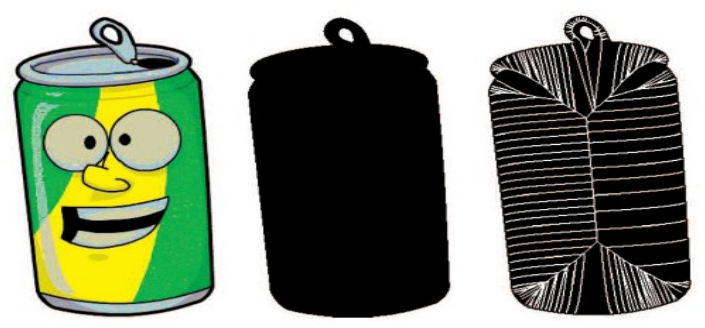

Fig. 2: Initial extracted skeleton with the additional branches

contour [16]. Skeleton achieved by the morphological operator method has many additional branches. In the method [17] the shortest distance from the boundary is firstly found for all points inside the shape. Points which have the longest distance from the boundary form the structure. In this research, maximum circles model is used for obtaining the skeleton. By this method, skeleton is obtained properly for the shapes that don't have any hole. Therefore, the shape's holes are filled by the shape filler filter before processing. Points which form the shape and its boundary are shown respectively by $D$ and $\partial D$. Having set of points $D$, the initial skeleton is calculated in the figure (2). In this way, for each point $D$, the maximum circle which has at least two points in the tangent on $\partial D$ and is located inside 
the shape is obtained. It is observed that the obtained skeleton has the additional branches which are omitted through the contour segmentation by DCE method. The next section describes additional branches omission in order to achieve a better skeleton.

\subsection{Skeleton pruning through the contour segmentation by DCE method}

The achieved skeleton is usually seen as noisy branches which are better to be omitted in the pre-processing step. This operation is called pruning. Therefore, in order to omit the additional branches of the skeleton, the boundary contour is segmented by DCE method $[21,19,18]$. Connecting the beginning and end of each section, an appropriate pruned structure is resulted, branches of which are thoroughly omitted. Suppose that $D$ is a set of the shape points and $\partial D$ is a set of the boundary points. Skeleton point $s$ is center of a circle which is cogent on $\partial D$ at least in two points. These points which are located in $\partial D$ and create the point $s$ are called $s$ creating points and are shown by $\tan (s) . S(D)$ is set of skeleton forming points. Without losing information, contour of each shape can be supposed as a polygon with lots of vertex. In fact, each point of the central contour is considered as a vertex. Numbers of sides are decreased in each step. Suppose that $s_{1}$ and $s_{2}$ are two consecutive sides of a polygon. $s_{1}$ and $s_{2}$ are replaced by one side. In a way, for all consecutive sides, the value $k$ is obtained in each step through the equation (1). Two consecutive sides which have the minimum value of $k$ are replaced by one side. Thus, numbers of the polygon's sides are decreased in each step.

$$
k\left(s_{1}, s_{2}\right)=\frac{\beta\left(s_{1}, s_{2}\right) l\left(s_{1}\right) l\left(s_{2}\right)}{l\left(s_{1}\right)+l\left(s_{2}\right)}
$$

In equation (1), $\beta$ is the angle between two sides $s_{1}$ and $s_{2^{\times}} l\left(s_{1}\right)$ and $l\left(s_{2}\right)$ are also side lengths of $s_{1}$ and $s_{2}$ respectively. Therefore, according to equation (1) it is expected that points with more convexity are selected as sides of the polygon. Having the polygon $p$ related to the boundary contour $\partial D$ which has $v$ peaks, polygon sides reduction is done as $p=p^{n}{ }_{s} p^{n-1}{ }_{s} p^{n-2}{ }_{p \times s} p^{a}$ by omitting the vertex $v$ of the polygon $P^{n-k}$ to achieve the polygon $P^{n-(k+1)}$. Figure (3) shows vertex omission steps by DCE method. It seems that the more sides are omitted, the less additional branches of the structure are. By default, side omission continues to reach a triangle. To stop the omission operation, stop parameter $D_{a v}\left(P^{n-k}\right)$ is defined. $D_{a v}\left(P^{n-k}\right)$ is average of distance between points of polygon $p$ and contour polygon $P^{n-k}$. Selecting the threshold of $K$, if $D_{a v}\left(P^{n-k}\right)>K$, side omission is stopped. Value of $K$ selection depends on the application. Having skeleton points $S(D)$ of the shape $D$ and contour polygon $p_{k}$, skeleton is pruned by omitting all points $s \in S(D)$ which are points creating $s$ in one section of the contour. In this case, $s$ is considered

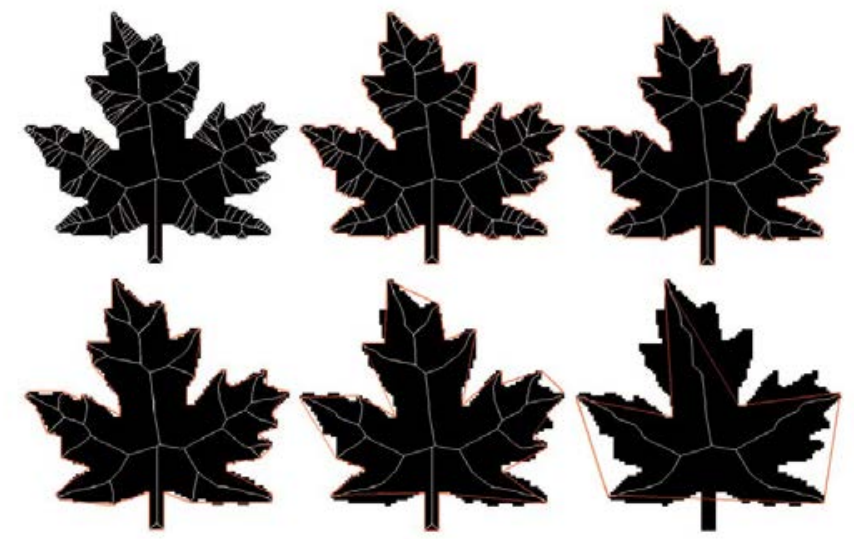

Fig. 3: Pruning the skeleton through contour segmentation by DCE method. Skeleton' branches are reduced by reduction of the polygon’s sides' number 

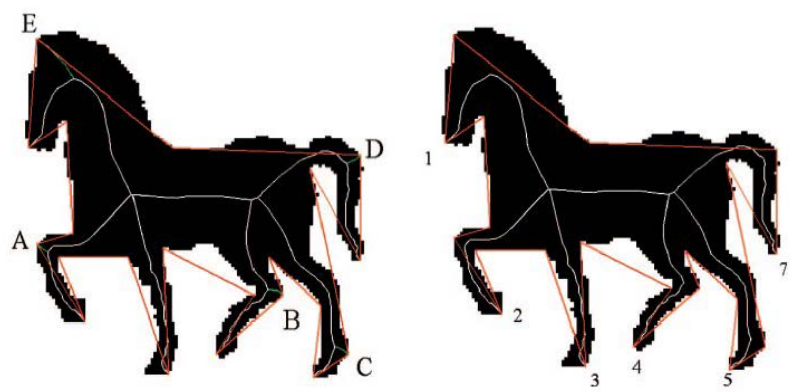

Fig. 4: On the left: structure has unimportant branches. On the right: unimportant branches omission

as an unimportant point and is omitted from $S(D)$. In figure (4), the skeleton have 5 branches and the final point of each branch is vertex of the polygon. In the DCE method, the obtained vertexes are the convex points of the contour. So many branches are omitted by this method but some of which are also remained. Another criterion is selected for omitting these branches. To do this, for each vertex $v$, distance $D_{1}(v)$ is calculated. $D_{1}(v)$ is the Euclidean distance between the point $v$ and the nearest concave point $u$ provided that the line $w v$ is located inside the shape. Threshold value of $T$ is here selected to omit the vertex $v$ for $D_{1}(v)<T$. Figure (6) shows vertex omission with the small value of $D_{1}(v)$. It is clear that a better skeleton is reached by omitting these branches.

\subsection{Feature extraction}

In order to find the corresponding points in two shapes, features of the points should be extracted. Consider two shapes $P$ and $Q$. The border contour's points are firstly calculated. Suppose that $p=\left\{p_{1}, p_{2}, \ldots \ldots p_{n}\right\}$ and $q=\left\{q_{1}, q_{2}, \ldots, q_{n}\right\}$ are respectively points set of border contour in shapes $P$ and $Q$ and also $S D_{1}$ and $S D_{2}$ are respectively pruned structure graph of shapes $P$ and $Q$. For each border contour's points, the shortest distance from the relevant structure is calculated. For example, the minimum distance of the point $p_{1}$ from the structure graph $S D_{1}$ is calculated and is shown by $w_{p 1}$. These calculations are done for all points of $p$ and $q$. Suppose that $w_{p}=\left\{w_{p 1}, w_{p 2}, \ldots, w_{p n}\right\}$ and $w_{q}=\left\{w_{q 1}, w_{q 2}, \ldots, w_{q n}\right\}$ are respectively Euclidean distance attributed to set $p$ and $q$. Set $w_{p}$ and $w_{q}$ are features extracted for points set of the border contour in $p$ and $q$ and matching is done according to proposed algorithm.
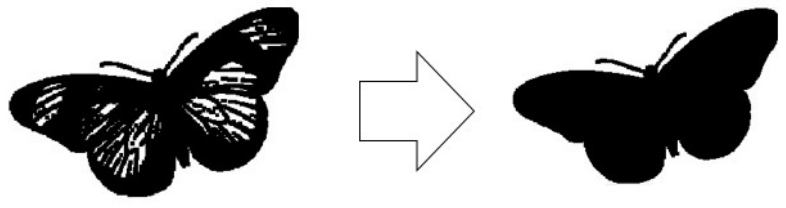

Fig. 5: Filling holes with the shape filler filter
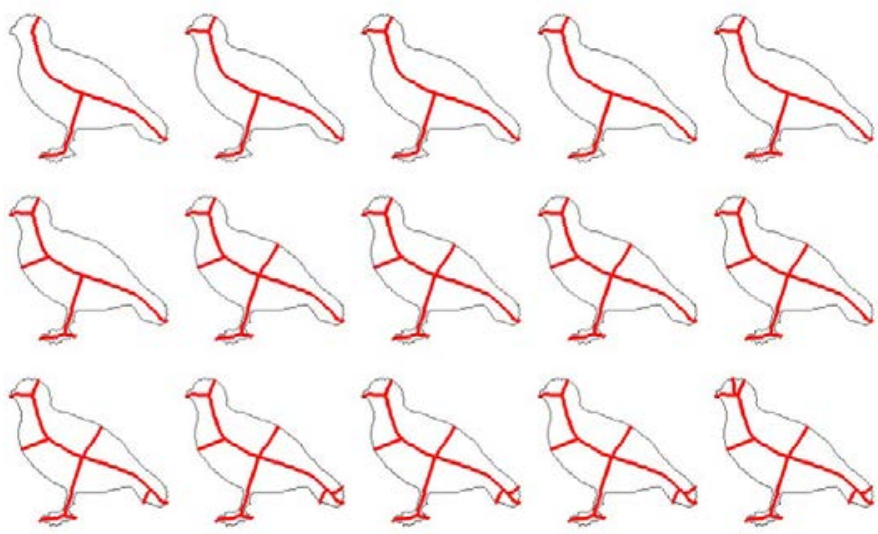
Fig. 6: Pruned skeleton for different values of $k$

\subsection{Matching}

In this section, corresponding points of sets $p=\left\{p_{1}, p_{2}, \ldots \ldots p_{n}\right\}$ and $q=\left\{q_{1}, q_{2}, \ldots, q_{n}\right\}$ are calculated according to the extracted feature. Suppose that point $p_{\mathrm{i}}$ corresponds with the point $q_{j}$. It is expected that difference between $w_{\mathrm{pi}}$ and $w_{\text {qj }}$ is small because distance of points from the structure is almost constant under the articulated changes. Equation (2) is obtained on this basis. It is firstly supposed that set $p=\left\{p_{1}, p_{2}, \ldots \ldots p_{n}\right\}$ correspond to set $q=\left\{q_{1}, q_{2}, \ldots, q_{n}\right\}$. Thus, $p_{1}$ corresponds with $q_{1}, p_{2}$ corresponds with $q_{2}$ and $p_{n}$ corresponds with $q_{n}$. Equation (2) is then calculated for $k=0$. After that, points set $q$ is shifted one position left. Suppose that $p=\left\{p_{1}, p_{2}, \ldots \ldots p_{n}\right\}$ correspond to $q=\left\{q_{2}, q_{2}, \ldots, q_{n}, q_{1}\right\}$ and accordingly, equation (2) is calculated for all rotations of $q$. Proper matching corresponds with lowest value of $\mathrm{C}$.

$$
C=\sum_{\mathrm{i}=1}^{n}\left|w_{p_{\mathrm{i}}}-w_{q_{\mathrm{i}+\mathrm{k}}}\right|^{-\underline{E}} \quad k=1,2, \ldots n
$$

\section{Implementation results}

Effectiveness of the proposed method for shape matching is evaluated in this section. Tests consist of two parts: at first, the pruned structure of the shape is extracted and then, corresponding points are calculated. In the preprocessing, the shape holes are firstly filled by the shape filler filter (figure 5). Structure is firstly calculated for each shape, after that its additional branches are pruned. Amount of the prunes branches depends on the stop parameter $\mathrm{K}$ selection. Figure (6) shows the pruned structure for different values of the stop parameter K. It is observed that if $\mathrm{K}$ value is low, main branches of the structure are omitted. In case the large amount of $\mathrm{K}$ is selected, the structure would have many noisy branches. Therefore, proper selection of $\mathrm{K}$ is significantly important in obtaining the corresponding points. In figure (7), respectively from right to left columns, the pruned structure with the proper $\mathrm{K}$ selection, minimum distance diagram of border contour's points from the structure, and the corresponding points are shown. Figure (7) shows matching of another shape. Proper amount of the corresponding points varies by selecting different values of $\mathrm{K}$. Increase in $\mathrm{K}$ value results in matching error increase, figure (9).
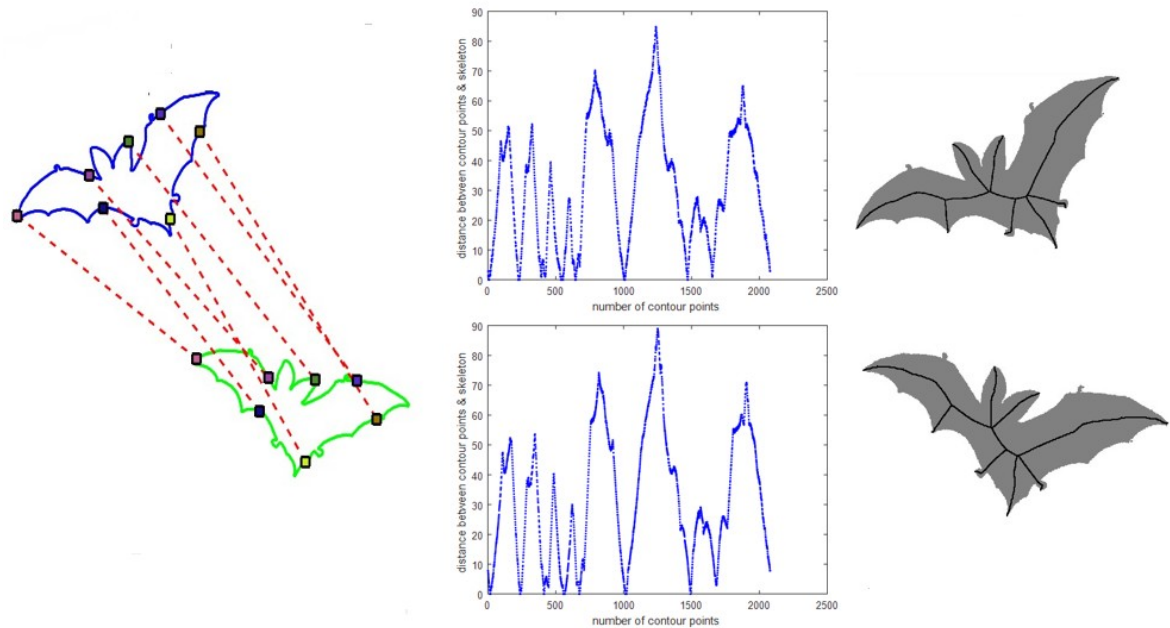

Fig. 7: Results of matching two bats; the first column from the right: pruned structure extraction, the middle column: minimum distance diagram of contour's points from the structure, the last column: finding the corresponding points. 

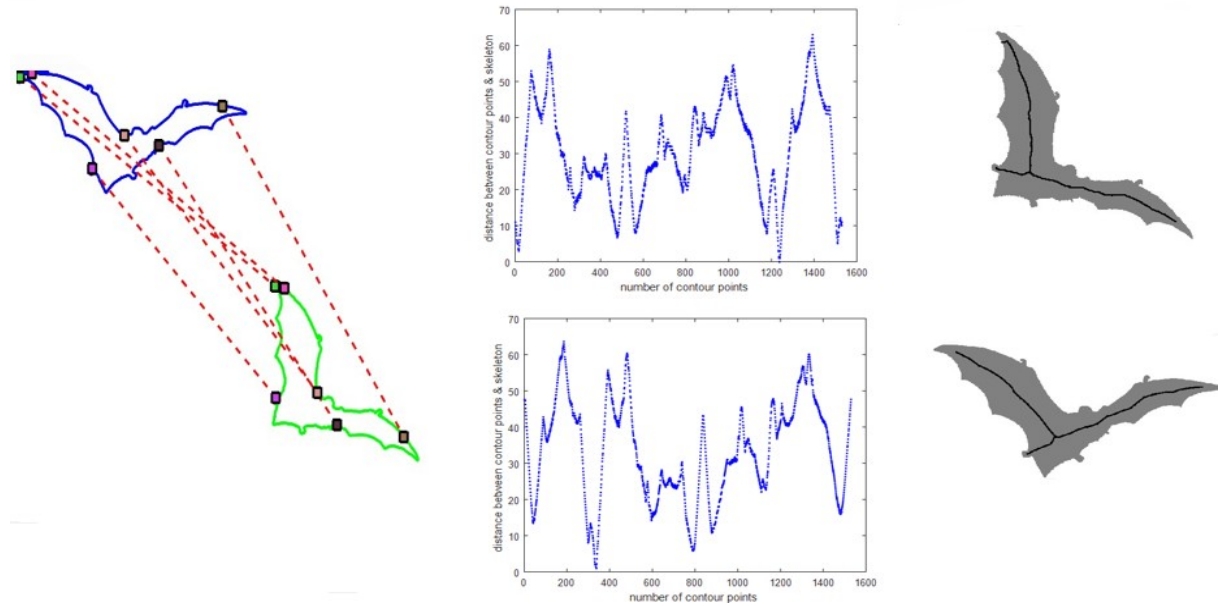

Fig. 8: Results of matching two birds

\section{Conclusion}

In this paper, a new algorithm is suggested for the shape matching which, unlike the method offered in [1,9], matching is done for all points of the contour. Some research does matching just for the points sampled from contour for matching error. For doing matching, shape structure is used as it consists of both geometrical and topologic features. Matching depends to a large extent on the extracted structure. The more accurate is the extracted structure, the better matching is resulted. When the structure is obtained, additional branches of which, with the shape contour segmentation, are omitted by DCE method. After that, points matching, which is the main section of this paper is

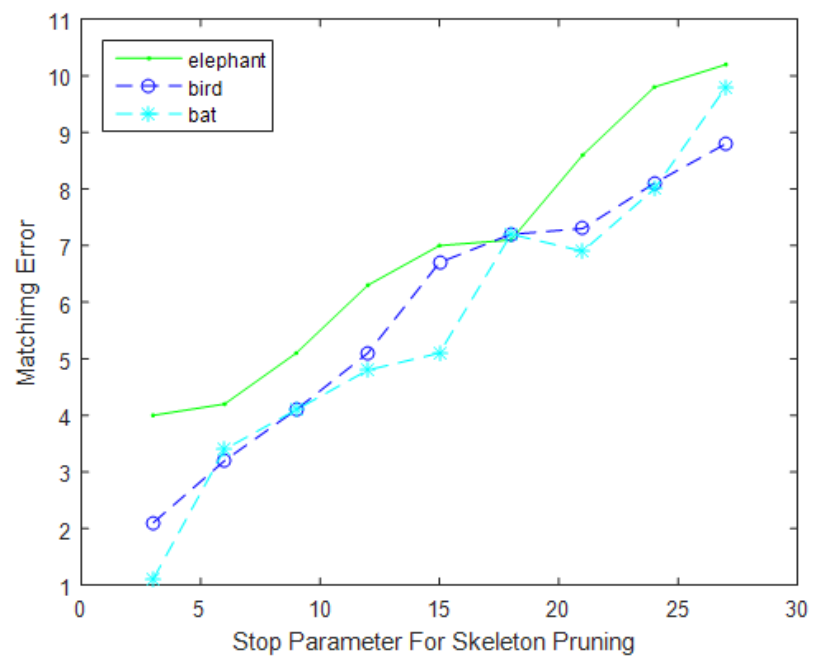

Fig. 9: Matching error by increasing the stop parameter $\mathrm{K}$ for three shapes of elephant, bird and bat

carried out through two steps. In the first step, minimum distance of all points of the shape border contour from the structure is calculated. It is expected that this distance is remained constant under the shape changes. In the next step, total differences of point distance in the first shape contour from the point distance in the second shape contour are calculated. Proper matching corresponds with the lowest value of this function. At the end, the algorithm is applied on the data base MPEG7 [24] and it shows the matching results. Matching is done with very high accuracy. It is worth mentioning that this method is applicable to the shapes which have the closed contour. On the other hand, such shapes mustn't have any hole. Matching shapes with the open contour and holes needs more research. 


\section{References}

[1] H. Chui and A. Rangarajan, "A new point matching algorithm for non-rigid registration," Computer Vision and Image Understanding, vol. 89, pp. 114-141, 2003.

[2] A. Torsello and E. R. Hancock, "A skeletal measure of 2D shape similarity," Computer Vision and Image Understanding, vol. 95, pp. 1-29, 2004.

[3] P. F. Felzenszwalb and J. D. Schwartz, "Hierarchical matching of deformable shapes," in Computer Vision and Pattern Recognition, 2007. CVPR'07. IEEE Conference on, 2007, pp. 1-8.

[4] Y. Gdalyahu and D. Weinshall, "Flexible syntactic matching of curves and its application to automatic hierarchical classification of silhouettes," Pattern Analysis and Machine Intelligence, IEEE Transactions on, vol. 21, pp. 1312-1328, 1999.

[5] E. G. Petrakis, A. Diplaros, and E. Milios, "Matching and retrieval of distorted and occluded shapes using dynamic programming," Pattern Analysis and Machine Intelligence, IEEE Transactions on, vol. 24, pp. 1501-1516, 2002.

[6] R. H. Davies, C. J. Twining, T. F. Cootes, J. C. Waterton, and C. J. Taylor, "A minimum description length approach to statistical shape modeling," Medical Imaging, IEEE Transactions on, vol. 21, pp. 525-537, 2002

[7] A. Thayananthan, B. Stenger, P. H. Torr, and R. Cipolla, "Shape context and chamfer matching in cluttered scenes," in Computer Vision and Pattern Recognition, 2003. Proceedings. 2003 IEEE Computer Society Conference on, 2003, pp. I-127-I-133 vol.1

[8] Z. Tu and A. L. Yuille, "Shape matching and recognition-using generative models and informative features," in Computer Vision-ECCV 2004, ed: Springer, 2004, pp. 195-209.

[9] H. Ling and D. W. Jacobs, "Using the inner-distance for classification of articulated shapes," in Computer Vision and Pattern Recognition, 2005. CVPR 2005. IEEE Computer Society Conference on, 2005, pp. 719-726.

[10] T. Sebastian, P. Klein, and B. Kimia, "Recognition of shapes by editing shock graphs," in null, 2001, p. 755.

[11] M. Cui, J. Femiani, J. Hu, P. Wonka, and A. Razdan, "Curve matching for open 2D curves," Pattern Recognition Letters, vol. 30, pp. 1-10, 2009.

[12] T.-L. Liu and D. Geiger, "Approximate tree matching and shape similarity," in Computer Vision, 1999. The Proceedings of the Seventh IEEE International Conference on, 1999, pp. 456-462.

[13] D. Sharvit ,J. Chan, H. Tek, and B. B. Kimia, "Symmetry-based indexing of image databases," in Content-Based Access of Image and Video Libraries, 1998. Proceedings. IEEE Workshop on, 1998, pp. 56-62.

[14] K. Siddiqi, S. Bouix, A. Tannenbaum, and S. W. Zucker, "Hamilton-jacobi skeletons," International Journal of Computer Vision, vol. 48, pp. 215-231, 2002.

[15] J. Xie, P.-A. Heng, and M. Shah, "Shape matching and modeling using skeletal context," Pattern Recognition, vol. 41, pp. 1756-1767, 2008.

[16] X. Bai and L .J. Latecki, "Path similarity skeleton graph matching," Pattern Analysis and Machine Intelligence, IEEE Transactions on, vol. 30, pp. 1282-1292, 2008.

[17] C. Yang, O. Tiebe, K. Shirahama, and M. Grzegorzek, "Object matching with hierarchical skeletons ",Pattern Recognition, vol. 55, pp. 183-197, 2016.

[18] A. Leborgne, J. Mille, and L. Tougne, "Noise-resistant Digital Euclidean Connected Skeleton for graph-based shape matching," Journal of Visual Communication and Image Representation, vol. 31, pp. 165-1.

[19] P. Golland, W. Eric, and L. Grimson, "Fixed topology skeletons," in Computer Vision and Pattern Recognition, 2000. Proceedings. IEEE Conference on, 2000, pp. 10-17.

[20] R. Kimmel, D. Shaked, N. Kiryati, and A. M. Bruckstein, "Skeletonization via distance maps and level sets," Computer vision and image understanding, vol. 62, pp. 382-391, 1995.

[21] C. M. Gold, D. Thibault, and Z. Liu, "Map generalization by skeleton retraction," in ICA Workshop on Map Generalization, Ottawa (August 1999.

[22] L. J. Latecki and R. Lakämper, "Convexity rule for shape decomposition based on discrete contour evolution," Computer Vision and Image Understanding, vol. 73, pp. 441-454, 1999.

[23] L. J. Latecki and R. Lakämper, "Polygon evolution by vertex deletion," in Scale-Space Theories in Computer Vision, ed: Springer, 1999, pp. 398-409.

[24] L. J. Latecki and R. Lakämper, "Application of planar shape comparison to object retrieval in image databases," Pattern Recognition, vol. 35, pp. 15-29, 2002. 